\title{
Supresión de anuncios de contactos sexuales: ¿autorregulación, legislación o colaboración institucional? Aproximación al contexto de Andalucía
}

\author{
The suppression of sexual contact advertisements: Self \\ regulation, legislation or institutional collaboration? An \\ approximation to the context of Andalusia
}

\author{
Fernando Martínez-Vallvey; Andrés Mellado-Segado
}

Cómo citar este artículo:

Martínez-Vallvey, Fernando; Mellado-Segado, Andrés (2019). "Supresión de anuncios de contactos sexuales: ¿autorregulación, legislación o colaboración institucional? Aproximación al contexto de Andalucía". El profesional de la información, v. 28, n. 2, e280223.

https://doi.org/10.3145/epi.2019.mar.23

Artículo recibido el 09-10-2018

Aceptación definitiva: 11-01-2019

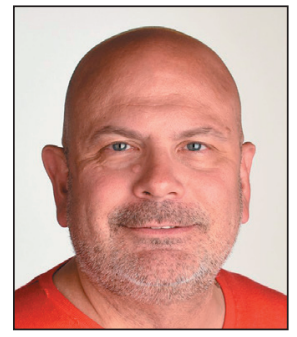

Fernando-María Martínez-

Vallvey $₫$

https://orcid.org/0000-0001-7686-1323

Universidad Pontificia de Salamanca

Henry Collet, 90-98. 37002 Salamanca,

España

fmvallvey@gmail.com

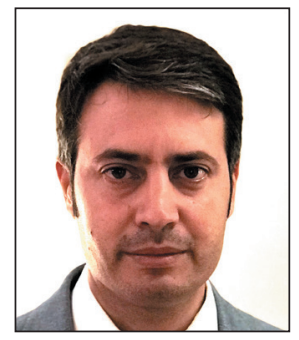

Andrés Mellado-Segado

https://orcid.org/0000-0002-2669-160X

Consejería de Educación de la Junta de Andalucía

EADE-University of Wales Trinity Saint David UWTSD

Juan Antonio de Vizarrón, s/n, Isla de la

Cartuja. 41092 Sevilla, España

andresmellado@yahoo.es

\section{Resumen}

Las dos principales fuentes de financiación de la prensa son las ventas de ejemplares al público y la venta de espacios publicitarios a los anunciantes. Un tipo específico de publicidad es la denominada "anuncios por palabras", entre los que se encuentran los anuncios de "contactos sexuales". En los últimos años su presencia en los diarios de España ha disminuido, a pesar de la falta de códigos éticos que limiten su presencia en las páginas y a que los diferentes gobiernos no han podido acometer una legislación que impida su publicación. El respeto a los derechos humanos de las mujeres y la lucha contra la trata y explotación de estas obligan a que esta práctica desaparezca, a pesar de la pérdida de ingresos que pueda suponer para una empresa periodística.

\section{Palabras clave}

Prensa diaria; Diarios; Contactos sexuales; Anuncios por palabras; Anuncios de contactos; Publicidad; Deontología; Ética; Economía de los medios; Igualdad de género; Dignidad; Prostitución; Autorregulación; Junta de Andalucía.

\begin{abstract}
Newspapers publish advertisements, by word count, that include ads for sexual encounters. In recent years the presence of these ads in the newspapers of Sevilla (Spain) have declined, in spite of a lack of ethical codes and government legislation that could completely prevent their publication. Respect for women's human rights, the fight against trafficking, and the exploitation of women means that this practice must disappear, despite the lost income for the journalistic enterprise.
\end{abstract}

\section{Keywords}

Daily press; Newspapers; Sexual encounters; Advertising; Advertisements; Classifieds; Deontology; Ethics; Economy of the media; Gender equality; Dignity; Sexual exploitation; Prostitution; Self-regulation; Junta de Andalucía; Andalusian regional government. 


\section{Introducción}

Las actividades ilegales en España suponen un 0,87\% del Producto Interior Bruto (PIB), según el Instituto Nacional de Estadística. La prostitución representa un $0,35 \%$ de dicho PIB, lo que implica aproximadamente 3.700 millones de euros. Asimismo el Ministerio de Interior calculó en 13.879 el número de personas en situación de riesgo por explotación sexual en 2015. El año anterior, el Centro de Inteligencia contra el Terrorismo y el Crimen Organizado detectó que había un total de 13.983 personas en riesgo de encontrarse en situación de trata de seres humanos y de explotación sexual (en 2013 la cifra era de 13.159 , lo que supuso un crecimiento de 824 , el $6,3 \%$ ). Como puede comprobarse, la cifra oscila entre 13.000 y 14.000 personas en España.

\section{El Plan integral de lucha contra la trata de mujeres y niñas con fines de explotación sexual 2015-2018 recuerda que}

"los medios de comunicación, escritos y audiovisuales, desempeñan un papel esencial no sólo desde el punto de vista de la información que proveen, sino en relación con el lucro obtenido de anuncios de contactos" (Ministerio de Sanidad, Igualdad y Servicios Sociales, s.f., p. 11).

Por ello convendría preguntarse ¿en qué medida los medios de comunicación pueden ser agentes proactivos para acabar con este tipo de violencia? ¿Los ingresos publicitarios justifican la difusión de anuncios que pueden encubrir situaciones de explotación sexual o de trata con fines de explotación sexual? ¿Es suficiente el autocontrol de las empresas periodísticas o habría que apostar por la vía legislativa?

En el ámbito académico se ha puesto de manifiesto la situación de estos anuncios. Ramón Reig apuntaba:

"Los mensajes no periodísticos en los medios incluso llevan a cabo una apología de la prostitución. Es significativo que se proyecte publicidad desde hace muchos años acerca de una actividad ilegal, por el momento, con la vista gorda de las autoridades. Como señal positiva, el hecho de que algunos medios se hayan negado en los últimos años a seguir con esa práctica" (Reig, 2015, pp. 97-98).

Los estudios sobre anuncios de contactos sexuales se han centrado en tres aspectos:

- sobre su legalidad o no en España: Megías-Quirós (2013), Balaguer-Callejón (2011), Martín-Morales (2011). Salvador-Coderch y Rubí-Puig (2010) realizaron un estudio de derecho comparativo entre Estados Unidos (en concreto el Estado de Nevada), Reino Unido y Alemania, al término del cual se analiza la situación en España;

- estudios que abordan una dimensión lingüística como Meneses-Falcón, Uroz-Olivares y Rúa-Vieite (2017), y Griffith et al. (2016).

- estudios sobre el contenido de estos anuncios, donde pueden destacarse los trabajos de Gutiérrez-García (2012), Torres-Díaz (2012), Rodríguez-Borges (2015) o Rodríguez-Borges y Torrado-Martín-Palomino (2017).

De otros países se pueden destacar los trabajos de Street y Norma (2016) y Szablewska y Kubacki (2018).

La mayoría de estudios sobre la prostitución insisten en los problemas sociales que genera. Otro aspecto tratado es la cosificación de la mujer en la publicidad (Alario-Gavilán, 2018; Vicente-Collado, 2009).

En cuanto a la situación legal de la publicación de los anuncios en varios países, la diversidad de comportamientos muestra la complejidad del tema. En algunos países, como Bélgica o Israel, está prohibida la publicación de anuncios que promuevan la prostitución (ProCon, 2018). Otros como Estados Unidos,

"sancionan la persuasión, la procuración y la promoción de la prostitución con fuertes multas y penas de prisión", a excepción del estado de Nevada (Global News, 2013).

En el caso del estado de Queensland, Australia (Queensland Government, 2018), se establece un formulario específico para la publicación de anuncios de contactos (Guidelines about the approved form for advertisements for prostitution) ${ }^{1}$.

Sobre el Reino Unido resulta interesante conocer las directrices de The Crown Prosecution Service con respecto a estos anuncios. En concreto, aconsejan a los periódicos el rechazo de su publicación:

"Si bien no existe un delito específico, Newspaper Society ha aconsejado a los editores que no publiquen anuncios de establecimientos ilegales como burdeles o la oferta ilegal de servicios sexuales. El Consejo también advierte a los editores de que los salones de masajes pueden ocultar ofertas ilegales de servicios sexuales y sugiere adoptar políticas de protección como las verificaciones de calificaciones para garantizar que el servicio anunciado sea legítimo. Se aconseja que una empresa periodística adopte la política de rechazar todos los anuncios de servicios personales, u otras políticas destinadas a reducir el riesgo de publicación relacionada con la prostitución ilegal y la trata de personas. La Guidance pide que las empresas periodísticas se aseguren de que su personal recibe asistencia y apoyo para tomar las decisiones de rechazar este tipo de publicidad o rechazar cualquier anuncio en particular. En algunas circunstancias, el propio periódico puede ser procesado por delitos de lavado de dinero en virtud de la Ley sobre el producto del delito de 2002" (CPS, 2018).

Estas cuestiones comportan un debate ético y deontológico en la profesión periodística y en el modelo de negocio de las empresas informativas. Una reflexión que se traslada a la esfera pública con el Informe de la ponencia sobre la prostitución en nuestro país, aprobada el 13 de marzo de 2007 por la Comisión Mixta de los Derechos de la Mujer y de la Igualdad 
de Oportunidades. En él se advertía de que la

"prostitución tiene una estrecha relación con los efectos negativos de los medios de comunicación" (Cortes Generales, 2007, pág. 44).

En la ponencia de esta Comisión Mixta también se incluía entre las posibles medidas de sensibilización, la renuncia a la publicidad relacionada con los contactos sexuales para impedir el negocio de las organizaciones mafiosas dedicadas al comercio sexual. Se realizaba una estimación del volumen de beneficios que generaba la prostitución en los medios vía ingresos publicitarios. En el periódico El país era de cinco millones de euros anuales (Cortes Generales, 2007, pág. 48). En este sentido, el periódico digital El confidencial afirmaba el 26 de febrero de 2007 que dos medios de referencia como El país y El mundo ingresaban más de 15.000 euros diarios por los anuncios de prostitución (Garrido, 2007).

El Estudio sobre la trata, la prostitución y otras formas de explotación en la ciudad de Sevilla (Ayuntamiento de Sevilla, 2013, p. 15) reconocía la existencia de un uso continuado de los anuncios de contactos, aunque ya presentaba una dinámica decreciente. Se contabilizaron en dos semanas de 2007 casi 3.000 anuncios en los medios de referencia de prensa escrita de pago. En cuanto al beneficio generado, según una estimación por tarifas publicitarias, rondaba los 1.982 euros en El país y 4.000 en El mundo-Estadio deportivo.

Dentro de esta sensibilización social, la Junta de Andalucía ha promovido la firma de un protocolo titulado Compromiso de buenas prácticas publicitarias en medios de comunicación de Andalucía, que se firmó el 14 de marzo de 2018. Participaron en el mismo una veintena de empresas de comunicación: ABC Sevilla, Grupo Joly, Diario de Cádiz, Diario Sur, Ideal de Granada, La voz de Almería, Diario de Jaén, Diario Córdoba, La razón, El mundo/Andalucía, El correo de Andalucía, Publicaciones del Sur, El desmarque, 20 minutos, Eldiario.es/Andalucía, El confidencial, Andalucesdiario.es, El plural, RTVA y Canal Sur radio y televisión, Cadena SER Andalucía, Atresmedia Radio, COPE Andalucía y la Asociación de Operadores de Telecomunicaciones Locales (Acutel).

Hasta llegar a este punto, de única aplicación a la comunidad andaluza, se plantearon en todo el Estado español diversas propuestas, algunas de las cuales no cuajaron. El debate sobre este tipo de anuncios por palabras se ha movido entre dos aguas, como tantas otras veces en los medios de comunicación: imposición externa o autorregulación.

En este artículo se repasa la discusión mediática, política y social en torno al problema de la publicación de los anuncios de contactos, y se analiza su publicación en la prensa sevillana, como paradigma de la prensa andaluza, a partir del debate político y social existente sobre estos temas.

\section{Prohibición por ley o autorregulación de la publicidad de contactos}

La Ley orgánica 1/2015, de 30 de marzo, aborda en el Capítulo V los delitos relativos a la prostitución y a la explotación sexual y corrupción de menores. Se establece que

"el que, empleando violencia, intimidación o engaño, o abusando de una situación de superioridad o de necesidad o vulnerabilidad de la víctima, determine a una persona mayor de edad a ejercer o a mantenerse en la prostitución, será castigado con penas..." (España, 2015).

Con esta redacción puede entenderse (Pérez-Vaquero, 2011) que la prostitución ejercida de forma libre y por una persona mayor de edad está despenalizada.

A partir de este punto, el debate se centra en los diferentes modos de abordar la prostitución por parte de la sociedad y de las autoridades. Barriga y Trujillo (2003) recogen las cuatro principales:

- prohibicionista

- abolicionista

- regulacionista

- garantista.

Otro enfoque es el relativo a la dignidad de las personas. Algunos autores (Prieto-Álvarez, 2005) consideran que el artículo 10 de la Constitución no se aplicaría al caso de la prostitución. Otros como Rey-Martínez, Mata-Martín y Serrano-Argüello (2004) plantean el abolicionismo de la prostitución y la necesidad de superar la pobreza de las prostitutas a través de otros trabajos y evitando la integración de la prostitución en mundo laboral. Salvador-Coderch y Rubí-Puig rechazan que:

"el Derecho -mucho menos el Derecho Constitucional- sea ontológicamente una realidad normativa distinta y al margen de la realidad fáctica. Por ello, queremos muy modestamente llamar la atención sobre la circunstancia de que, normativamente es perfectamente defendible sostener posiciones abolicionistas, pero que la ilegalización no seguida de aplicación efectiva del Derecho o, mucho peor, la alegalización de un entorno de relaciones jurídicas de prestación de servicios -los sexuales, en nuestro caso- puede acabar resultando de hecho mucho más degradante para la condición humana de las personas afectadas que una regulación limitativa y rigurosa de tal actividad" (Salvador-Coderch; Rubí-Puig, 2010, p. 137). 
Estos autores apuntan que entre los criterios que podrían utilizar los legisladores para prohibir la publicidad de este tipo de anuncios se encuentra el desvalor que históricamente ha acompañado a la prostitución, a lo que se suma la protección de la infancia y la juventud, la salvaguarda de la libertad individual y de la integridad de las personas víctimas de la explotación sexual.

El debate sobre la presencia de los anuncios de contactos sexuales se mueve entre dos extremos. Por una parte, libertad de empresa, libertad de expresión, prohibición de publicitar una actividad que no es ilegal², la asfixia presupuestaria por la crisis económica, el deseo de autorregulación por parte de cada medio de comunicación..., frente a otras realidades como mujer-mercancía, explotación, vulneración de derechos fundamentales, peligro para la integridad de los menores, prohibición de inversión publicitaria con dinero público, etc.

En este marco de antagonismos se incluyen algunos argumentos que tanto las empresas informativas como los poderes del Estado han esgrimido en el debate sobre la legitimidad de publicar o prohibir la publicidad de anuncios relacionados con la prostitución. Dos posturas históricamente encontradas y situadas en un punto muerto en el que los medios de comunicación amagan con la retirada de los anuncios de "contactos" (algunos medios lo realizaron definitivamente por iniciativa propia y otros porque la publicidad ha derivado hacia otros canales como internet) y los distintos gobiernos recomiendan su eliminación sin mayor fuerza coercitiva. ¿Qué pasos se han dado en el siglo XXI en este debate?

Desde que en 2005 se produjera la primera denuncia de un particular sobre los anuncios de contactos, se han sucedido numerosas aportaciones. Entre ellas, la ponencia de la Comisión en el Congreso de los Diputados, la inclusión de la problemática en los distintos planes integrales contra la Trata de seres humanos con fines de explotación sexual o dos proposiciones de ley impulsadas por el Bloque $\mathrm{Na}$ cionalista Gallego y Unión del Pueblo Navarro, así como un informe del Consejo de Estado. También en el Debate sobre el estado de la nación en julio de 2010 se anunció que se trabajaba para eliminar los anuncios y algunos grupos pidieron como medida de presión que no se in-

\section{En el Debate sobre el estado de la nación de 2010 algunos grupos políticos pidie- ron que las empresas periodísticas que publicaran estos anuncios no recibieran publicidad institucional}

cluyese en la planificación publicitaria de la comunicación institucional a las empresas periodísticas que publicaran este tipo de anuncios.

En ese año el Grupo Parlamentario Socialista registró una proposición no de ley titulada Sobre la publicidad de la prostitución en medios de comunicación, en la que se consideraba que

"para eliminar este tráfico de personas y facilitar la reintegración de las explotadas sexualmente destaca algo que puede parecer obvio pero que en ocasiones se olvida: es imperativo reducir la demanda".

En definitiva, planteaban al Gobierno que se fomentara

"la autorregulación dentro de la prensa escrita encaminada a eliminar la publicidad de mujeres prostituidas. El establecimiento por parte de las administraciones públicas de las medidas oportunas que promuevan la desaparición de los anuncios de prostitución".

En esta proposición no de ley se reconocía el problema de legislar sólo contra los anuncios en prensa escrita:

"Pero no sólo podemos hablar de los medios escritos, ya que vemos una creciente presencia de estos anuncios en televisión o en internet, por lo que serían necesarias herramientas más elaboradas y avanzadas que simplemente su prohibición por ley".

En este período se han pronunciado varios líderes de opinión y asociaciones con diversos intereses sociales como la Asociación de Editores de Diarios Españoles (AEDE), que rechazó la iniciativa y mostró su indignación pues

"si la prostitución no estaba prohibida, ¿por qué iba a estar prohibida su publicidad?" (Recogido en el reportaje

“Negocio alegal, anuncio ilegal” de El país del 23 de marzo de 2011) (Gómez; Morán-Breña, 2011).

Sin embargo, no todos los medios escritos tenían este planteamiento. Por ejemplo, el diario Público fue uno de los primeros en retirar los anuncios de contactos sexuales y dotarse de un manual interno sobre cómo abordar la violencia de género. Javier Salas firmaba en este diario un texto titulado "Los anuncios de prostitución ya son ilegales" el 18 de julio de 2010 (Salas, 2010). Tres años antes, Ignacio Escolar había realizado una apuesta por combatir el proxenetismo en un texto titulado "En Público no somos proxenetas", publicado el 7 de octubre de 2007. Recordaba en su blog que

"No somos el primer periódico que toma esta decisión, aunque sí el primero que nace sin estos anuncios y el primer diario de pago en España. Antes que nosotros, el gratuito 20 minutos también renunció a esta publicidad. Fuera de España, algunas grandes cabeceras, como el International herald tribune, han dejado de publicar estos módulos tras descubrirse que detrás de ellos, en algunas ocasiones, se esconden redes mafiosas, trata de blancas, tráfico de personas. Esclavitud" (Escolar-García, 2007). 
Tiene especial relevancia el informe del Consejo de Estado titulado Informe sobre las posibilidades de actuación contra anuncios de contenido sexual y prostitución publicados a diario en diversos medios de comunicación de prensa escrita (Consejo de Estado, 2010). Fue encargado por la entonces ministra de Igualdad, Bibiana Aido, para avalar o descartar la iniciativa de prohibir los anuncios. Fundamentó la petición de prohibición de los anuncios en que contienen imágenes y mensajes que atentan contra la dignidad de la mujer cuyos servicios ofrecen, implican un trato vejatorio y degradante, vulneran el valor fundamental de la igualdad entre mujeres y hombres, y pueden favorecer la transmisión de roles y estereotipos contrarios a dichos derechos y valores, y favorecedores de la violencia de género, por cuanto transmiten la idea de un género femenino sumiso, subordinado, servil e inferior" (Consejo de Estado, 2011, p. 3).

Además, los contenidos gráficos contribuyen a que perviva la idea de mujer como mero objeto; este tipo de publicidad es contraria a los derechos de la infancia y la juventud; transmite roles y estereotipos de género perpetuadores de desigualdad y subordinación, y pueden, en ocasiones, dar carta de naturaleza a una actividad delictiva como el proxenetismo.

En sesión celebrada el 9 de marzo de 2011, la Comisión de Estudios de este organismo abogaba por "una norma legal distinta de la Ley general de publicidad" (Consejo de Estado, 2011, p. 55) que permitiese acotar con exactitud y claridad el ámbito subjetivo y objetivo de la prohibición. Reforzaba así la idea de la regulación normativa en detrimento de la autorregulación.

Por su parte, el Consejo de Estado (2011, p. 57) amplió el foco a otros medios de difusión como las televisiones e internet, considerando en el primer caso una prohibición expresa de este tipo de anuncios, tanto en horario de protección de menores como en otros más amplios. En el caso de internet, señalaba la necesidad de sopesar la pertinencia de establecer algún tipo de limitación (Consejo de Estado, 2011, p. 59).

El Gobierno de España no se sumó a todos los puntos del informe del Consejo de Estado. Valoró el respaldo a la prohibición de los anuncios de prostitución en la prensa escrita, pero la fórmula para acometerla no sería una normativa específica, sino la modificación del artículo 8 de la Ley general de publicidad, mediante la inclusión de un nuevo apartado en el que se pretendía prohibir la publicidad en prensa de servicios sexuales y de locales dedicados a la prostitución, alcanzando la misma a las ediciones digitales de estas publicaciones. De esta forma se seguiría una actuación similar a la que se plantea con materiales y productos sanitarios, los que puedan generar riesgos para la salud o la seguridad de las personas, los juegos de azar, las bebidas alcohólicas de más de 20 grados y el tabaco. Recordemos que hay más prohibiciones publicitarias en diferentes ámbitos, como en la de emisión de publicidad de partidos políticos en campañas electorales (Artículo 60 de la Ley orgánica 5/1985 de Régimen electoral general). Nunca ha llegado a modificarse la Ley general de publicidad en ese sentido.

Por otra parte, tanto los editores de prensa escrita como la Asociación de Usuarios de la Comunicación (AUC) defendieron la autorregulación a través de códigos éticos, como una manera de evitar la publicidad de estos anuncios. La AUC se mostraba abiertamente partidaria de la corregulación:

“En la línea ya existente para los servicios telefónicos de tarificación adicional: un marco legal regulatorio, un código deontológico complementario y una aplicación ad casum del mismo en una comisión en la que participarán editores, organizaciones sociales y la propia Administración" (AUC, 2010, p. 8).

El problema radica en determinar qué organismo "regula esa autorregulación". Por ejemplo, se había comprobado que el "jurado de la publicidad" no entraba en el fondo de esta práctica comercial. La Asociación de Usuarios de la Comunicación sólo contemplaba una serie de recomendaciones en su informe de 2010 y los comportamientos sobre este asunto quedaban al arbitrio de cada medio. Ni siquiera existía un código ético al que pudieran adscribirse los medios de comunicación.

\section{El Consejo de Estado en su informe (Consejo de Estado, 2011, p. 31) manifestaba que este sistema regulatorio}

"se ha mostrado insuficiente para alcanzar la finalidad perseguida de hacer desaparecer los anuncios de la prostitución en la prensa escrita",

pero sirvió de vía intermedia entre la eliminación que unos propugnaban y el derecho a incluir esta modalidad publicitaria por parte de los medios de comunicación. Una salida que enfrió el debate que presentaba, como contexto, la crisis económica que afectaba a los medios, que ya habían visto reducir sus ingresos al aplicarse otras prohibiciones como, por ejemplo, publicitar el consumo de tabaco. 
Las últimas referencias también inciden en la autorregulación. Así el Plan integral de lucha contra la trata de mujeres y niñas con fines de explotación sexual 2015-2018 establece en su objetivo número 13 la

"promoción de la suscripción de códigos de autorregulación que favorezcan la eliminación de anuncios de contacto en los medios de comunicación e impulso de una comisión para el estudio de fórmulas tendentes a su eliminación" (Ministerio de Sanidad, Igualdad y Servicios Sociales, s.f.).

Asimismo, la Consejería de Igualdad y Políticas Sociales (Junta de Andalucía, 2015, 23 de septiembre) anunció el I Plan integral para la erradicación de la prostitución y la trata de mujeres y niñas con fines de explotación sexual en Andalucía que incluirá, según fuentes del Instituto Andaluz de la Mujer, un apartado de "tolerancia cero" a este tipo de anuncios de contactos (Junta de Andalucía, 2015).

En este contexto cabe destacar la iniciativa de la Junta de Andalucía para erradicar los anuncios de contactos. Una veintena de periódicos firmaron un compromiso de buenas prácticas publicitarias, denominado Compromiso de buenas prácticas publicitarias en medios de comunicación de Andalucía. Este convenio está en línea con el Convenio del Consejo de Europa sobre prevención y lucha contra la violencia contra la mujer y la violencia doméstica, ratificado en Estambul el 11 de mayo de 2011 (España, 2014). La presidenta de la Junta de Andalucía, Susana Díaz, consideró que el Compromiso firmado el 14 de marzo de 2018 es

"un ejemplo de compromiso y responsabilidad social (que camina) hacia los valores que este país necesita".

El objetivo del Compromiso es

"hacer de Andalucía un espacio libre de mensajes de contenidos publicitarios de índole sexual ligados a los denominados 'anuncios de contacto', conscientes de que constituyen la punta del iceberg de situaciones graves que atentan contra los derechos, la integridad y la dignidad de las personas, fundamentalmente mujeres y niñas, como la prostitución, la esclavitud sexual o la trata" (Junta de Andalucía, 2018).

Cada parte firmante asume un papel para la erradicación de la violencia contra las mujeres en forma de explotación sexual:

"Si a los poderes ejecutivo y legislativo corresponde la revisión del marco jurídico y su adecuación a la nueva realidad que debe ordenar, a los medios de comunicación les compete una labor de sensibilización de la sociedad desde la indiscutible posición que desempeñan en el desarrollo de la libertad de expresión y del derecho a la información" (Junta de Andalucía, 2018).

Esta actuación de la Junta a través del Compromiso podría encajar en las funciones simbólicas que los Estados pueden y deben desarrollar, junto con las coercitivas, recordando la idea de Locke, sobre la diferencia entre la capacidad imperativa o persuasiva del Estado. Recoge Garton-Ash que:

"mientras el poder coercitivo del Estado debe usarse con precaución extrema en lo relativo a la libertad de expresión [dentro de la que se incluye la publicidad] sobran los motivos para que el Estado emplee su poder simbólico para lo que él [Corey Brettschneider] Ilama 'persuasión democrática'. Así, por ejemplo, el Estado puede 'hablar' mediante la designación de un aniversario como un Día del Holocausto o de Martin Luther King Jr., mediante monumentos y museos, a través de declaraciones del Parlamento y del presidente y, lo que es quizá más importante, a través de lo que se imparte en la enseñanza reglada por el Estado" (Garton-Ash, 2017, p. 126).

Esta idea de la capacidad persuasiva y didáctica del Estado, en este caso del gobierno autonómico andaluz, puede ayudar a sortear los problemas jurídicos puestos de relieve desde organismos como el Consejo de Estado para suprimir los anuncios de contactos en la prensa. Una sociedad que desee erradicar una práctica tan violenta y explotadora contra las mujeres realiza una acción ejemplificadora con medidas como la del Compromiso, sin tener que entrar en el complicado mundo de la legislación sobre libertad de expresión.

\section{Anuncios clasificados en el mercado de la prostitución}

En este apartado analizaremos la evolución de la presencia de los anuncios de contactos sexuales en la prensa sevillana. Para ello utilizaremos datos aportados por dos documentos públicos: el Informe de la ponencia sobre la prostitución en nuestro país (Cortes Generales, 2007) y Estudio sobre la trata, la prostitución y otras formas de explotación sexual en la ciudad de Sevilla (Ayuntamiento de Sevilla, 2013). Los datos relacionados con los años 2016 (del 9 al 16 de octubre) y 2018 (del 16 al 23 de julio, toda vez que había pasado un tiempo prudencial desde la firma del Compromiso de buenas prácticas publicitarias con la Junta de Andalucía) se han obtenido directamente por los autores de este trabajo. De esta manera se pueden hacer comparaciones y establecer una evolución de los anuncios de sexo desde 2007, con los datos que ofrecían las dos ponencias citadas. 
La primera referencia que podemos tomar sobre la presencia de anuncios de publicidad sexual en la prensa escrita se incluye en el Informe de la ponencia sobre la prostitución en nuestro país (Cortes Generales, 2007) donde se señala que en un período de catorce días las ediciones de la prensa publicaban 5.608 anuncios. En una cata realizada expresamente para esa ponencia, ABC publicaba 2.969; El mundo, 730; El país, 480; y La razón, 251.

En el Estudio sobre la trata, la prostitución y otras formas de explotación sexual en la ciudad de Sevilla elaborado seis años después, en 2013, por el Ayuntamiento de Sevilla, se recogen las siguientes cifras:

"A lo largo de las dos semanas de recogida de datos en la prensa, el total de anuncios analizados ha sido de 2.935. Ordenándolos en función del número de anuncios, encontramos que el periódico que más publicidad incluye es Estadio deportivo -suplemento deportivo del diario El mundo- con el 38,4\% del total. El 26,8\% de los anuncios recogidos son del $A B C$, El país reúne el 15,8\% del total de los anuncios, el Diario de Sevilla el 13,4\%, el Correo de Andalucía el 5,1\% y sólo el 0,5\% corresponden al periódico deportivo AS. La prensa gratuita 20 minutos y Viva Sevilla no han presentado ningún anuncio de prostitución u otras formas de sexo comercial" (Ayuntamiento de Sevilla, 2013).

Desglosado en datos absolutos, las cifras son:

- Estado deportivo/El mundo: 1.126

- ABC, 788

- El país, 463

- Diario de Sevilla: 395

- El correo de Andalucía: 149

- As: 14

La razón ya había tomado la decisión de no publicar este tipo de anuncios. El 23 de diciembre de 2009 el periódico del Grupo Planeta decidía poner fin a este tipo de publicidad, decisión que, para el director de La razón, Francisco Marhuenda, fue un "un orgullo" pues "son vejatorios, no tienen nada que ver con la publicidad" (Aguirre, 2011).

Entre 2007 y 2013 se produjeron reducciones considerables. La razón, del cien por cien; ABC, del 50 por ciento; El mundo había desplazado estos anuncios a su suplemento deportivo; mientras que El país, Diario de Sevilla y El correo de Andalucía se mantenían en cantidades similares.

En 2016, en la medición realizada por los autores de este artículo, se observa otro considerable descenso en el número de anuncios de contenido sexual. Nuestra observación siguió el mismo parámetro que ambos estudios del Ayuntamiento de Sevilla de 2007 y 2013 (citados en Ayuntamiento de Sevilla, 2013, p. 16): contar el número de anuncios en dos semanas, en este caso la segunda quincena de octubre. Así se mantiene la ausencia de anuncios en La razón y El mundo (que había dejado de editar el suplemento deportivo). El diario ABC publicó 19; el Diario de Sevilla, 73; y El país, 320.

Como puede verse El país mantiene en esa fecha la primera posición en cantidad de anuncios de contactos. En ese año se editaba en Andalucía la edición nacional, y la sección de "Clasificados. Adultos" se incluía en las páginas de "Pantallas", en un faldón donde se reproduce la programación de televisión o en la sección de deportes.

Todo ello a pesar de que el presidente del consejo de administración de Prisa, Juan Luis Cebrián, afirmara que se planteaba prescindir de la publicidad de la prostitución, pero que no estaba decidido:

"Cualquier exceso de puritanismo me parece mal por parte de las fuerzas políticas en ese tema, porque afecta a la felicidad y a la vida de muchas personas, que se ven marginadas precisamente por el puritanismo y por la corrección política" (Cebrián, 2010, p. 64).

El caso de El correo de Andalucía presentaba una particularidad especial. Había dejado de anunciar servicios de prostitución para publicitar los servicios de una agencia matrimonial para "personas singles, altamente motivadas en buscar pareja y nuevas amistades" (unici.es). Se trata de dar difusión pagada a un negocio legal de personas que libremente consienten utilizar un servicio determinado. En este caso se considera que no se trata de una publicidad vejatoria o discriminatoria, que no atenta contra derechos fundamentales.

En cuanto a El mundo (edición de Andalucía), la retirada comportaba razones éticas, informativas y económicas. El entonces director Francisco Rossel decidió poner fin a esta sección. Una decisión en la que también influyó el menor número de páginas para la edición Andalucía y la retirada progresiva de las ediciones locales "en papel", que provocaba a su vez menos espacio para el contenido informativo. Además, según sus responsables comerciales era una sección con poco rédito económico por lo que se procedió a eliminarla.

El ejemplo más claro de los anuncios analizados en la segunda quincena de octubre de 2016 que representa a la mujer-mercancía, a la mujer objeto sexual como reclamo, era Diario de Sevilla. En su edición diaria mantenía anuncios con fotografías de contenido pornográfico utilizando la imagen de la mujer como objeto de deseo.
El Grupo Joly, consciente del debate social y moral de la publicación de los anuncios de contactos sexuales, decidió retirarlos desde el 1 de noviembre de 2016 
En el Decálogo para una publicidad no sexista del Instituto Andaluz de la Mujer se afirmaba que se presenta el cuerpo femenino como

"objeto desvinculado del producto que se pretende publicitar con el fin de atraer la atención de su potencial clientela" (Junta de Andalucía, s.f., punto 6).

Sin embargo, el Grupo Joly, consciente del debate social y moral de la publicación de los anuncios de contactos sexuales, decidió retirarlos desde el 1 de noviembre de 2016. A partir de esa fecha ya no están incluidos en Diario de Sevilla y Málaga hoy, y han ido desapareciendo paulatinamente del resto de cabeceras en Andalucía.

En el último análisis de anuncios realizado por los autores de este trabajo, en la semana del 16 al 23 de julio del 2018 , se ha detectado que, salvo el diario $A B C$, ninguno de los medios analizados conserva la sección o espacio de anuncios por palabras, lo que refuerza la idea de que eran los anuncios de contactos los que mantenían esta página en los diarios. Sólo sobreviven anuncios de empresas inmobiliarias y las esquelas. En el caso de $A B C$, los anuncios apenas ocupan una página y se centran en Inmobiliarias, Belleza y un genérico Diversos, que no incluye anuncios de contactos. En esta misma quincena se han analizado las ediciones nacionales de los diarios $A B C, E l$ mundo y El país. El único que mantiene una pequeñísima sección de anuncios por palabras, apenas un faldón, es El mundo. En esa sección no se incluyen anuncios de contactos. $A B C$ y El país ya no incluyen los anuncios al haber eliminado la sección.

Para el caso andaluz, puede considerarse que la firma del Compromiso de buenas prácticas, promovido por la Junta de Andalucía, presidida en la fecha de la publicación por una mujer ${ }^{3}$, Susana Díaz, y suscrito por una veintena de diarios ha surtido efecto y los anuncios de contactos han desaparecido de las páginas de los diarios. El Compromiso no tiene rango de ley, pero puede valorarse como una medida política que ejemplifica también el poder de las acciones conjuntas entre los órganos de gobierno y la sociedad civil.

\section{Conclusiones}

La publicación de anuncios de contactos en la prensa diaria se encamina a su desaparición. Tiene una difusión casi residual y cada vez es mayor el número de medios de comunicación escrita que han descartado su difusión frente a los que la mantienen, fundamentalmente por fines estrictamente comerciales.

En el caso sevillano la disminución fue progresiva, pasando de 137 anuncios diarios a 47 primero, y a su desaparición actual en la prensa andaluza. Este comportamiento, promovido por el gobierno autonómico y secundado por las empresas periodísticas, puede servir de ejemplo para otras comunidades autónomas o para toda España.

Sin embargo estamos ante una problemática con más peso cualitativo que cuantitativo. Independientemente del número de anuncios hay que centrar el foco en:

- la complicidad involuntaria que pueden mantener los medios que continúen publicando estos anuncios de contactos con la vulneración de derechos fundamentales;

- la posibilidad de un atentado contra la dignidad de las personas;

- en cooperar en que se produzca un trato denigrante y vejatorio;

- que pueda facilitarse la trata de personas y a que las mafias utilicen la publicidad como fuente de enriquecimiento ilícito.

La crisis económica ha golpeado duramente la cuenta de resultados de las empresas informativas y el recorte de algunas vías de ingresos plantea dificultades a la hora de tomar decisiones tendentes a renunciar a otros. Ahora bien, no puede ser considerada una coartada para el mantenimiento de estos anuncios. Urge actuar para elaborar códigos éticos, como en Bélgica o Israel, que eliminen esta práctica comunicativa. Instituciones y empresas informativas, a través de fórmulas participadas, han de adquirir un compromiso real con la problemática de la publicidad de la prostitución.

El marco actual de autorregulación se consideró “insuficiente” por parte del Consejo de Estado en 2009 y desde entonces poco se ha avanzado. Una vez adormecido el debate público y mediático no se tomaron decisiones legales y algunas publicaciones siguen incluyendo anuncios clasificados. Las entidades que tienen que velar por el comportamiento ético de la publicidad tampoco aportaron soluciones prácticas.

Todos los agentes implicados hacen un buen diagnóstico de la situación y de las consecuencias de la trata de personas, de cómo la publicidad puede propiciar situaciones de esclavitud sexual, pero ninguno ofrece soluciones ni por la vía normativa ni por el autocontrol de las empresas periodísticas. En este sentido, parece que el Compromiso promovido por la Junta de Andalucía y firmado por una veintena de periódicos y empresas periodísticas ha dado un resultado positivo para erradicar en la prensa diaria andaluza este tipo de anuncios. Cada día son más los diarios del resto de España que están suprimiendo estos anuncios, como La gaceta regional de Salamanca, donde diariamente podían encontrarse dos o tres columnas de "contactos" (en el mes de junio de 2018 apenas llegaban a la cuarentena); y que a partir del 1 de enero de 2019 ha dejado de publicarlos gracias a un acuerdo firmado con el Ayuntamiento salamantino. Una política de "compromisos" puede haber servido para sortear los problemas legales o las dificultades de autorregulación. 


\section{Notas}

1. En algunos casos, las prostitutas han denunciado que la prohibición de algunos términos resulta "absurda". Véase: Brook, 2018. Todo ello evidencia la complejidad de este problema.

2. Puede recordarse en este punto la polémica decisión de agosto de 2018 para la aprobación del sindicato Otras, que conllevó la dimisión de la directora general de Trabajo, Concepción Pascual Lizana. Las actividades relacionadas con el sexo no son ilegales, por ejemplo, las actividades de los actores del cine pornográfico. Sin embargo, la ministra de Trabajo Magdalena Valerio mostró su contrariedad ante esta decisión, porque el ejecutivo socialista mantiene una postura abolicionista frente a la prostitución. En concreto aseguró:

"No avalaremos un sindicato de una actividad que no es legal y que vulnera los derechos de las mujeres. No lo hará un Gobierno socialista y feminista".

El sindicato Otras fue aprobado y publicado en el BOE porque la tramitación cumplía todos los requisitos legales relacionados con la Ley orgánica de libertad sindical y administrativamente no había ningún inconveniente, aunque desde el Ministerio de Trabajo nadie actuó desde un plano político para impedir su legalización.

3. Aunque no ha habido ninguna mujer que haya alcanzado la presidencia del Gobierno de España, sí ha habido varias, de diferentes partidos políticos, que han presidido comunidades autónomas.

\section{Referencias}

Aguirre, Lilian (2011). “Francisco Marhuenda: 'Es un orgullo no publicar anuncios de prostitución'”. La razón, 4 octubre. http://www.larazon.es/historico/3902-francisco-marhuenda-critica-a-los-agregadores-de-contenidos-en-la-red-HLLA_ RAZON_402218\#.Ttt1WkddMuE7sj2

Alario-Gavilán, Mónica (2018). “La influencia del imaginario de la pornografía hegemónica en la construcción del deseo sexual masculino prostituyente: un análisis de la demanda de prostitución”. Asparkía, n. 33, pp. 61-79.

https://doi.org/10.6035/Asparkia.2018.33.4

AUC (2010). Los anuncios de contactos sexuales en la prensa. Consideración legal y propuesta de regulación. Asociación de Usuarios de la Comunicación.

http://www.auc.es

Ayuntamiento de Sevilla (2010). Plan de Acción Integral para promover la erradicación de la trata, la prostitución y otras formas de explotación sexual (2010-2015).

https://www.sevilla.org/servicios/mujer-igualdad/planes-municipales/plan-integral-contra-la-prostitucion/iplanintegral-prostitucion.pdf

Ayuntamiento de Sevilla (2013). Estudio sobre la trata, la prostitución y otras formas de explotación en la ciudad de Sevilla.

https://www.sevilla.org/servicios/mujer-igualdad/planes-municipales/plan-integral-contra-la-prostitucion/estudiosobre-la-trata-la-prostitucion-y-otras-formas-de-explotacion-sexual-en-la-ciudad-de-sevilla.pdf

Ayuntamiento de Sevilla (2016). II Plan de acción integral para luchar contra la trata, la prostitución y otras formas de explotación sexual del Ayuntamiento de Sevilla (2016-2020).

https://www.sevilla.org/servicios/mujer-igualdad/planes-municipales/plan-integral-contra-la-prostitucion/ii-plan-deaccion-integral-1.pdf

Balaguer-Callejón, María-Luisa (2011). "Anuncios de prostitución en la prensa escrita”. Artículo 14, una perspectiva de género: Boletín de información y análisis jurídico, n. 36, pp. 31-32.

Barriga, Silverio; Trujillo, Inmaculada (2003). "Prostitución: ¿libertad y esclavitud?”. Anduli. Revista andaluza de ciencias sociales, n. 3, pp. 95-111.

https://revistascientificas.us.es/index.php/anduli/article/view/3775

Basílio-de-Simões, Rita (2018). "News media power and public policy: The mediatized construction of women trafficking for sexual exploitation". Brazilian journalism research, v. 14, n. 1.

https://doi.org/10.25200/BJR.v14n1.2018.1039

Brook, Benedict (2018). "The 'nutty' list of words that Queensland sex workers aren't allowed to use in adverts". News. com.au, 20 April.

https://www.news.com.au/technology/online/censorship/the-nutty-list-of-words-that-queens/and-sex-workers-arentallowed-to-use-in-adverts/news-story/2eef1956f2759ce2f74cf55622eb33a5

Brufao-Curiel, Pedro (2008). Prostitución y políticas públicas: entre la reglamentación, la legalización y la abolición. Madrid: Fundación Alternativas. ISBN: 9788496653979

http://www.fundacionalternativas.com/public/storage/estudios_documentos_archivos/xmlimport-jPZHqj.pdf 
Cebrián, Juan-Luis (2010). "La brecha entre poder y ciudadanos”. En: Nueva Economía Fórum (ed.). La encrucijada de los medios de comunicación. Debates del curso 2009/2010. Madrid: Nueva Economía Fórum, SL; Fundación Bertelsmann, pp. 47-68. ISBN: 9788493763954

Consejo de Estado (2011). Informe sobre las posibilidades de actuación contra anuncios de contenido sexual y prostitución publicados a diario en diversos medios de comunicación de prensa escrita (№ E 1/2010).

http://www.consejo-estado.es/pdf/Anuncios\%20de\%20contenido\%20sexual\%20y\%20prostitucion\%20en\%20prensa.pdf

Cortes Generales (España) (2007). Informe de la ponencia sobre la prostitución en nuestro país (154/9). Cortes Generales: Comisión Mixta de los Derechos de la Mujer y de la Igualdad de Oportunidades.

http://www.lourdesmunozsantamaria.cat/IMG/pdf/INFORME_PONENCIA_PROSTITUCION.pdf

CPS (2018). Prostitution and exploitation of prostitution. The Crown Prosecution Services.

https://www.cps.gov.uk/legal-guidance/prostitution-and-exploitation-prostitution

Escolar-García, Ignacio (2007). “En Público no somos proxenetas”. Público, 7 octubre.

http://www.escolar.net/MT/archives/2007/10/en-publico-no-somos-proxenetas.html

España (2014). "Instrumento de ratificación del Convenio del Consejo de Europa sobre prevención y lucha contra la violencia contra la mujer y la violencia doméstica, hecho en Estambul el 11 de mayo de 2011". BOE, n. 137, 6 junio.

https://www.boe.es/diario_boe/txt.php?id=BOE-A-2014-5947

España (2015). “Ley orgánica 1/2015, de 30 de marzo, por la que se modifica la ley orgánica 10/1995, de 23 de noviembre, del Código penal". BOE, n. 77, 31 marzo.

https://www.boe.es/diario_boe/txt.php?id=BOE-A-2015-3439

Garrido, Óscar (2007). "El mundo y El país ingresan diariamente más de 15.000 euros por los anuncios de prostitución". El confidencial, 26 febrero.

https://www.elconfidencial.com/comunicacion/2007-02-26/el-mundo-y-el-pais-ingresan-diariamente-mas-de-15-000euros-por-los-anuncios-de-prostitucion_511490

Garton-Ash, Timothy (2017). Libertad de palabra. Diez principios para un mundo conectado. Barcelona: Tusquets. ISBN: 9788490663981

Global news (2013). "Prostitution laws around the world". Global news, 20 December. https://globalnews.ca/news/128029/at-a-glance-prostitution-laws

Gómez, Rosario G.; Morán-Breña, Carmen (2001). “Negocio alegal, anuncio ilegal”. El país, 23 marzo. https://elpais.com/diario/2011/03/23/sociedad/1300834801_850215.html

Griffith, James D.; Capiola, August; Balotti, Brandon; Hart, Christian L.; Turner, Ryan (2016). “Online female escort advertisements: The cost of sex". Evolutionary psychology, v. 14, n. 2.

https://doi.org/10.1177/1474704916651270

Gutiérrez-García, Andrea (2012). "Prostitución y trata de seres humanos con fines de explotación sexual: análisis de la situación actual a través de los anuncios de contactos". En: Vázquez-Bermúdez, Isabel (coord.). Investigación y género, inseparables en el presente y en el futuro. IV Congreso universitario nacional investigación y género. Sevilla: Universidad de Sevilla, pp. 829-842. ISBN 9788494897535 https://idus.us.es/xmlui/handle/11441/39943

Junta de Andalucía (s.f.). Decálogo para una publicidad no sexista. Junta de Andalucía. Consejería de Igualdad y Políticas Sociales. Instituto Andaluz de la Mujer.

http.//www.juntadeandalucia.es/institutodelamujer/catalogo/doc/iam/2015/143486445.pdf

Junta de Andalucía (2015). La Junta presenta las líneas estratégicas del Plan integral contra la prostitución y la trata con fines de explotación sexual. Junta de Andalucía. Consejería de Igualdad y Políticas Sociales, 23 de septiembre https://www.juntadeandalucia.es/organismos/igualdadypoliticassociales/actualidad/noticias/detalle/107873.html

Junta de Andalucía (2018). Compromiso de buenas prácticas publicitarias en medios de comunicación de Andalucía. https://bit.ly/2GINAz1

Martín-Morales, Ricardo (2011). "Prostitución, anuncios publicitarios y principios constitucionales". Teoría y realidad constitucional, n. 28, pp. 597-608.

https://doi.org/10.5944/trc.28.2011.6974

Megías-Quirós, José-Justo (2013). “Publicidad y prostitución”. Anuario de filosofía del derecho, v. 29, pp. 425-442. https://dialnet.unirioja.es/servlet/articulo?codigo $=4550660$ 
Meneses-Falcón, Carmen; Uroz-Olivares, Jorge; Rúa-Vieite, Antonio (2017). "Flyers y anuncios de servicios sexuales en Madrid". Revista latina de comunicación social, n. 72, pp. 145-164.

https://doi.org/10.4185/RLCS-2017-1158

Ministerio de Sanidad, Igualdad y Servicios Sociales (s.f.). Plan integral de lucha contra la trata de mujeres y niñas con fines de explotación sexual 2015-2018. Ministerio de Sanidad, Igualdad y Servicios Sociales. Secretaría de Estado de Servicios Sociales e Igualdad. Delegación del Gobierno para la Violencia de Género.

http://www.violenciagenero.igualdad.mpr.gob.es/planActuacion/planContraExplotacionSexual/docs/Plan_Integral_ Trata_18_Septiembre2015_2018.pdf

Pérez-Vaquero, Carlos (2011). “¿Legalizar la prostitución?”. Criminología y justicia, n. 1, septiembre-noviembre, p. 9. https://dialnet.unirioja.es/servlet/articulo?codigo=3886965

ProCon.org (2018). "100 countries and their prostitution policies". ProCon.org, 23 April. https://prostitution.procon.org/view.resource.php?resourcelD=000772

Prieto-Álvarez, Tomás (2005). La dignidad de la persona. Núcleo de la moralidad y el orden públicos, límite al ejercicio de las libertades públicas. Madrid: Civitas Ediciones. ISBN: 9788447023110

Queensland Government (2018). Guidelines about the Approved form for advertisements for prostitution, 6 August. http://www.pla.qld.gov.au/advertising/guidelinesApproveFormAdvertPros.htm

Reig, Ramón (2015). Crisis del sistema, crisis del periodismo. Contexto estructural y deseos de cambio. Barcelona: Gedisa. ISBN: 9788497849111

Rey-Martínez, Fernando; Mata-Martín, Ricardo M.; Serrano-Argüello, Noemí (2004). Prostitución y derecho. Madrid: Aranzadi. ISBN 8497678354

Rodríguez-Borges, Rodrigo-Fidel (2015). “Mujeres inmigrantes en los anuncios de prostitución de la prensa española. Un análisis documental”. En: García-Castaño, Francisco-Javier; Megías-Megías, Adelaida; Ortega-Torres, Jennifer (eds.). Actas del VIII Congreso sobre migraciones internacionales en España: Granada, 16-18 de septiembre de 2015. Granada: Universidad de Granada, Instituto de Migraciones, 398 pp. ISBN: 9788492139040 https://dialnet.unirioja.es/servlet/articulo?codigo $=6201439$

Rodríguez-Borges, Rodrigo-Fidel; Torrado-Martín-Palomino, Esther (2017). "Un estudio sobre el comercio sexual de mujeres y publicidad. El papel de la prensa española". Vivat academia. Revista de comunicación, n. 141, pp. 93-114. http://doi.org/10.15178/va.2017.141.93-114

Salas, Javier (2010). "Los anuncios de prostitución ya son ilegales”. Público, 18 julio. https://www.publico.es/espana/anuncios-prostitucion-ya-son-ilegales.html

Salvador-Coderch, Pablo; Rubí-Puig, Antonio (2010). "Libertad de expresión, discurso comercial y publicidad de prostitución". Revista española de derecho constitucional, n. 90, pp. 111-145.

https://dialnet.unirioja.es/servlet/articulo?codigo=3394905

Street, Tatum; Norma, Caroline (2016). "Sex tourists in their own country: Digital media advertising of Asian women by the Australian sex industry". Gender technology \& development, v. 20, n. 3, pp. 279-305.

https://doi.org/10.1177/0971852416667885

Szablewska, Natalia; Kubacki, Krzysztof (2018). “Anti-human trafficking campaigns: A systematic literature review”. Social marketing quarterly, v. 24, n. 2, pp. 104-122.

https://doi.org/10.1177/1524500418771611

Torres-Díaz, María-Concepción (2015). "Prensa escrita y anuncios de contacto ¿libertad sin igualdad? Análisis crítico desde un enfoque de género y constitucional". En: Suárez-Villegas, Juan-Carlos; Liberia-Vayá, Irene; Zurbano-Berenguer, Belén (eds.). I Congreso internacional de comunicación y género. Libro de actas: 5-7 de marzo de 2012. Sevilla: Facultad de Comunicación. Universidad de Sevilla, pp. 1089-1108. ISBN: 9788467679564

https://idus.us.es/xmlui/bitstream/handle/11441/38455/Pages\%20from\%20LIBRO\%20ACTAS\%20I\%20CONGRESO\%20 COMUNICACI\%C3\%93N\%20Y\%20G\%C3\%89NERO_2.pdf?sequence=1

Vicente-Collado, Sara (2009). “La prostitución también es violencia machista”. Crítica, v. 59, n. 960, pp. 48-52. https://dialnet.unirioja.es/servlet/articulo?codigo=2939374 